\title{
The development of loop-mediated isothermal amplification targeting alpha-tubulin DNA for the rapid detection of Plasmodium vivax
}

Sylvatrie-Danne Dinzouna-Boutamba' ${ }^{1}$, Hye-Won Yang ${ }^{1}$, So-Young Joo ${ }^{1}$, Sookwan Jeong ${ }^{2}$, Byoung-Kuk Na ${ }^{3}$, Noboru Inoue ${ }^{4}$, Won-Ki Lee ${ }^{5}$, Hyun-Hee Kong ${ }^{6}$, Dong-ll Chung ${ }^{1}$, Youn-Kyoung Goo ${ }^{1}$ and Yeonchul Hong ${ }^{1 *}$

\begin{abstract}
Background: Malaria that is caused by Plasmodium vivax is the most widely distributed human malaria. Its recent resurgence in many parts of the world, including the Republic of Korea (ROK), emphasizes the importance of improved access to the early and accurate detection of $P$. vivax to reduce disease burden. In this study, a rapid and efficient loop-mediated isothermal amplification (LAMP)-based method was developed and validated using blood samples from malaria-suspected patients.
\end{abstract}

Method: A LAMP assay targeting the a-tubulin gene for the detection of $P$. vivax was developed with six primers that recognize different regions of the target gene. The diagnostic performance of the a-tubulin LAMP assay was compared to three other tests: microscopic examinations, rapid diagnostic tests (RDTs), and nested polymerase chain reactions (PCRs) using 177 whole blood specimens obtained from ROK military personnel from May to December 2011.

Results: The a-tubulin LAMP assay was highly sensitive with a detection limit of 100 copies of $P$. vivax a-tubulin gene per reaction within 50 min. It specifically amplified the target gene only from $P$. vivax. Validation of the a-tubulin LAMP assay showed that the assay had the highest sensitivity $(P<0.001$ versus microscopy; $P=0.0023$ versus RDT) when nested PCR was used as the gold standard and better agreement (concordance: $94.9 \%$, kappa value: 0.865) with nested PCR than RDT and microscopy. A Receiver Operation Characteristics analysis showed that the diagnostic accuracy of the a-tubulin LAMP assay for vivax malaria was higher (Area Under Curve $=0.908$ ) than RDT and microscopy.

Conclusion: This study showed that the P. vivax a-tubulin LAMP assay, which can be used to diagnose early infections of vivax malaria, is an alternative molecular diagnostic tool and a point-of-care test that may help to prevent transmission in endemic areas.

Keywords: Malaria, Plasmodium vivax, Immunochromatographic rapid diagnostic tests, Polymerase chain reaction, Loop-mediated isothermal amplification

\footnotetext{
* Correspondence: ychong@knu.ac.kr

'Department of Parasitology and Tropical Medicine, Kyungpook National

University School of Medicine, 700-422 Daegu, Republic of Korea

Full list of author information is available at the end of the article
} 


\section{Background}

Plasmodium vivax, which is a causative agent of human malaria, is the most widely distributed species, and 2.8 billion people are at risk for transmission in the world [1]. Vivax malaria reemerged in 1993 in the Republic of Korea (ROK or South Korea), and its incidence rapidly increased to 4,142 by 2000 . Plasmodium vivax malaria is commonly believed to be clinically benign and selflimiting $[2,3]$. However, accumulating lines of evidence have shown that the impacts of $P$. vivax malaria with respect to economic and social burdens in endemic regions have been underestimated [4-6]. Moreover, the resurgence of vivax malaria in many parts of the world, including the ROK $[7,8]$, emphasizes the importance of improving access to reliable diagnostic methods that facilitate the early and accurate diagnosis of malaria, which is urgently required to facilitate disease management and control [9].

Microscopic examinations of Giemsa-stained thick and thin blood films, which are considered the gold standard for the diagnosis of malaria $[10,11]$, are recommended by the World Health Organization. Although this technique is highly specific, its sensitivity for the detection of $P$. vivax is lower than for Plasmodium falciparum due to the low parasitaemia of P. vivax [12]. Furthermore, it is time consuming, labour-intensive, and requires technical expertise with respect to the interpretation of Giemsastained blood smears. Immunochromatographic rapid diagnostic tests (RDTs) that are based on the detection of histidine-rich protein-2 and/or lactate dehydrogenase $[11,13]$ provide rapid and straightforward field tests for the detection of $P$. vivax but are limited in terms of sensitivity and specificity $[14,15]$. Accordingly, molecular diagnostic methods, such as polymerase chain reaction (PCR) and nested PCR, have been developed and used to improve $P$. vivax detection [16-21]. Although these assays have been shown to be highly effective for diagnosing malaria, they require laboratory equipment, trained personnel, and have long turnaround times, which limit their usefulness for routine diagnoses in the field [22].

Loop-mediated isothermal amplification (LAMP), which is a relatively straightforward and sensitive technique that is based on rapid DNA amplification under isothermal conditions, was recently developed to remove the need for sophisticated and expensive thermal cyclers [23]. LAMP involves the specific amplification of target DNA by Bacillus stearothermophilus (Bst) DNA polymerase, allowing strand displacement DNA synthesis with a set of six oligonucleotides that recognize independent regions of the target gene. The use of six oligonucleotides improves the specificity and speed of the amplification and forms a loop-structured amplicon, which produces a typical ladderpattern of multiple bands [24]. A positive reaction is easily determined by eye as turbidity [25] or fluorescence by the inclusion of fluorescent detection dyes, such as SYBR green or hydroxynaphthol under UV light [26,27]. These features allow LAMP assays to be used to detect many pathogenic organisms, such as viruses, bacteria, fungi, parasites, and vivax malaria parasites [28-31].

The present study was undertaken to develop an $\alpha$ tubulin targeting LAMP assay for the detection of $P$. vivax and to validate the assay using whole blood from suspected malaria patients. The sensitivity and specificity of the devised $P$. vivax $\alpha$-tubulin LAMP assay were determined and compared with those of microscopy and RDTs with $18 \mathrm{~S}$ ribosomal RNA (rRNA)-based nested PCR as gold standard. To validate the accuracies of the $\alpha$-tubulin targeting LAMP assay, the performances of the tests examined were assessed using receiver operating characteristic (ROC) [32,33].

\section{Methods}

\section{Samples}

This study was conducted at Armed Forces Hospitals that treat soldiers stationed near the DMZ, which separates the ROK from the Democratic People's Republic of Korea (DPRK or North Korea), in the northern part of the Gyeonggi-do Province, in the northwest region of the ROK (between $37^{\circ}-38^{\circ}$ latitude and $127^{\circ}-128^{\circ}$ longitude). This is a high-risk area for malaria and where only $P$. vivax is transmitted [34]. All enrolled soldiers had no history of travel to malaria-endemic areas and had never received a blood transfusion. Whole blood samples were collected by sequential sampling from 177 male ROK soldiers, who provided written informed consent, among all 189 ROK male soldiers who had been admitted to the Armed Forces Hospitals (from May to December 2011) with febrile illness (temperature $\geq 38^{\circ} \mathrm{C}$ ) and were clinically suspected to have malaria. To detect $P$. vivax, approximately $1 \mathrm{~mL}$ of blood was collected by venipuncture into a vacutainer tube (Becton Dickinson, Franklin Lakes, NJ, USA) containing ethylenediaminetetraacetic acid (EDTA). Samples were transported on ice within $4 \mathrm{~h}$ to a laboratory (Department of Parasitology and Tropical Medicine, Kyungpook National University School of Medicine, Daegu, ROK) where they were stored at $-70^{\circ} \mathrm{C}$. As is required by the Declaration of Helsinki, donor confidentiality was maintained throughout, and the study was approved by the Ethics Committee of the Armed Forces Medical Command (AFMC-13-IRB-053, July 2011).

\section{Microscopic examination and rapid diagnostic tests}

These samples were assayed using standard diagnostic procedures, including direct microscopic examination of Giemsa-stained thick and thin blood films and RDT. The 177 blood samples from malaria-suspected patients were first examined with RDT and microscopy at the Armed Forces Hospitals. The results for microscopy and 
RDT were recorded by two different technicians. Then, each sample was blinded and transported to the laboratory (Department of Parasitology and Tropical Medicine, Kyungpook National University School of Medicine, Daegu) for further microscopic examination. A slide was considered negative if no asexual stages of Plasmodium spp. were found during examination of 100 fields. Parasite densities were assessed by counting against 200 leucocytes, and converting to parasites per microliter, assuming a standard leucocyte count of $8,000 / \mu \mathrm{L}$. The immunochromatographic RDT (SD malaria Ag Pf/Pan,
Standard Diagnostic, Inc., Hagal-Dong, Korea) detects the parasite antigen Plasmodium falciparum Histidinerich protein-2 (PfHRP-2) specific to P. falciparum in one capture site and pan-Plasmodium lactate dehydrogenase (pan-pLDH) for all four Plasmodium species, in a separate capture line.

\section{a-tubulin LAMP assay}

The $P$. vivax-specific LAMP primers were designed based on previously described using the Primer Explorer program [23,35] (Figure 1). The $\alpha$-tubulin sequences of

\section{A} F3 F2

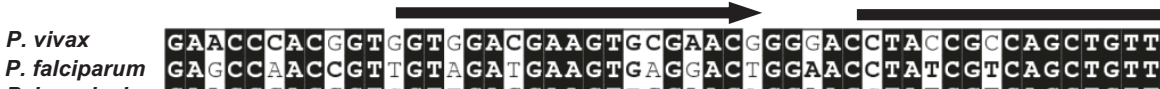

$\begin{array}{ll}\text { P. falciparum } & \text { GAGCCAACCGTTGTAGATGAAGTG AGGACT GAACCTATCGTCAGCTGTT } \\ P . \text { knowlesi } & \text { GAACCCACCGTCGTTGACGAAGTTCGAACAGGAACGTATCGTCAGCTGTT }\end{array}$

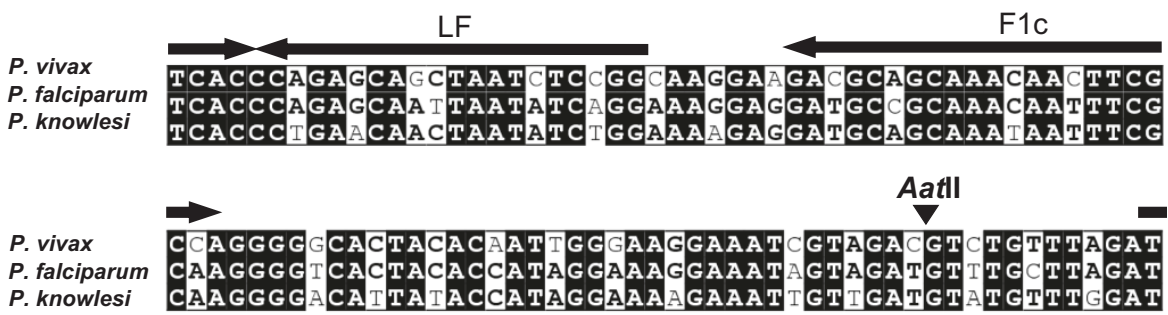

P. knowlesi CAAGGGGACATTA ACCATAGGAAAGAAAT TGTGATGTIGTTTGGAT

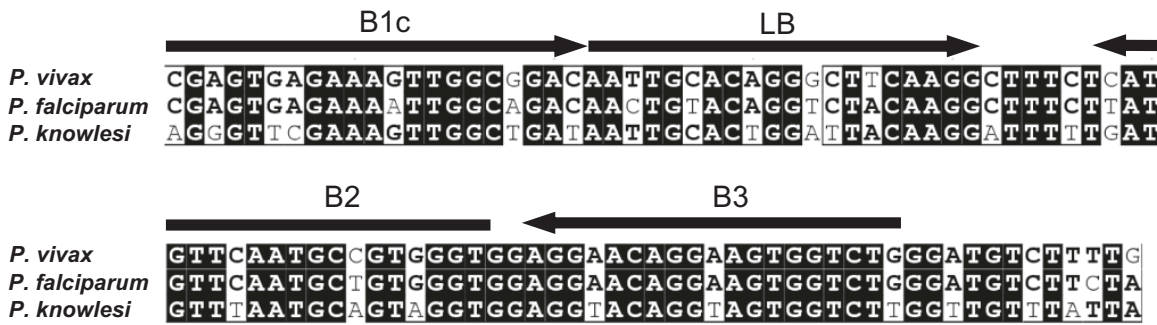

B

\begin{tabular}{ll}
\hline Primer & \multicolumn{1}{c}{ Sequence (5'- 3') } \\
\hline F3 & GTGGACGAAGTGCGAACG \\
B3 & CAGACCACTTCCTGTTCCTC \\
FIP (F1c-F2) & TGGCGAAGTTGTTTGCTGCGTCTACCGCCAGCTGTTTCAC \\
BIP (B1c-B2) & TCGAGTGAGAAAGTTGGCGGACACCCACGGCATTGAACATG \\
LF & CCGGAGATTAGCTGCTCTGG \\
LB & AATTGCACAGGGCTTCAAGG \\
\hline
\end{tabular}

Figure 1 Primer design for a-tubulin loop-mediated isothermal amplification (LAMP) assay for the detection of Plasmodium vivax. (A) The primers were selected based on a nucleotide sequence alignment of the target region of a-tubulin gene from P. vivax, P. falciparum [GenBank accession no. XM_001351490] and Plasmodium knowlesi [GenBank accession no. XM_002258237] using ClustalW software. Black boxes and bold fonts indicate identical and conserved nucleotides, respectively. The locations of the primer recognition sites are indicated by arrows with the primer names. The black arrowhead indicates the Aatll cleavage site. F3, forward outer primer; B3, backward outer primer; FIP, forward inner primer; BIP, backward inner primer; LF, loop forward primer; and LB, loop backward primer. (B) Sequences of the a-tubulin LAMP primers. 
$P$. vivax that were used for primer design were retrieved from GenBank [P. vivax, GenBank accession no. XM 001615073]. The $P$. vivax-specific $\alpha$-tubulin LAMP primer set consisted of F3 (forward outer primer), B3 (backward outer primer), FIP (forward inner primer), BIP (backward inner primer), LF (loop forward primer), and LB (loop backward primer). LAMP was performed for $90 \mathrm{~min}$ at $64^{\circ} \mathrm{C}$ in a $25-\mu \mathrm{L}$ mixture containing 40 pmol each of FIP and BIP, 5 pmol each of F3 and B3, 20 pmol each of LF and LB, $1.4 \mathrm{mM}$ deoxynucleoside triphosphates, $0.8 \mathrm{M}$ betaine, $1 \mu \mathrm{L}$ of Bst DNA polymerase (New England Biolabs, Ipswich, MA, USA) in $2.5 \mu \mathrm{L}$ of buffer $(20 \mathrm{mM}$ Tris- $\mathrm{HCl}, \mathrm{pH} 8.8 ; 10 \mathrm{mM}$ $\mathrm{KCl} ; 10 \mathrm{mM}\left(\mathrm{NH}_{4}\right)_{2} \mathrm{SO}_{4} ; 8 \mathrm{mM} \mathrm{MgSO}$; and $0.1 \%$ Tween 20), and $1 \mu \mathrm{L}$ of the plasmid containing the $\alpha$ tubulin gene fragment or genomic DNA from the whole blood of malaria-suspected patients in a Loopamp realtime turbidimeter (Realoop-30; Eiken Chemical Co., Ltd., Tokyo, Japan). After the LAMP assay, the reactions were inactivated for $2 \mathrm{~min}$ at $80^{\circ} \mathrm{C}$ and then evaluated by electrophoresis in an agarose gel $(2.0 \%)$ or visualized by a fluorescence detection reagent (FD; Eiken Chemical Co., Ltd.) under UV light. The $\alpha$-tubulin LAMP using DNA from patients was performed in duplicate. Results from the LAMP assay were independently recorded using the UV lamp in real-time turbidimeter (Eiken Chemical Co., Ltd.) by two investigators who were blinded to the identity of the samples (Table 1 and Table 2). If the results of the duplicate reactions were discrepant, a third amplification and detection were performed to resolve the discrepancy. After scoring the results of fluorescence detection, all the LAMP products were electrophoresed on a $2 \%$ agarose gels for detection of LAMP amplification. The results from gel electrophoresis were consistent with those from fluorescence detection.

Table 1 Comparison of the results obtained from the four diagnostic methods

\begin{tabular}{|c|c|c|c|c|}
\hline \multicolumn{4}{|c|}{ Diagnostic test results } & \multirow{2}{*}{$\begin{array}{l}\text { No. of } \\
\text { cases } \\
(\%)\end{array}$} \\
\hline Microscopy & RDT & Nested PCR & a-tubulin LAMP & \\
\hline+ & + & + & + & $96(54.2)$ \\
\hline- & + & + & + & $23(13.0)$ \\
\hline- & - & + & + & $5(2.8)$ \\
\hline- & - & + & + & $4(2.3)$ \\
\hline- & + & - & + & $8(3.4)$ \\
\hline- & + & - & - & $10(5.6)$ \\
\hline- & - & - & + & $1(0.6)$ \\
\hline \multirow[t]{2}{*}{-} & - & - & - & $30(16.9)$ \\
\hline & Total & & & 177 \\
\hline
\end{tabular}

\section{Analytical sensitivity and specificity of the a-tubulin LAMP assay}

The $\alpha$-tubulin gene was amplified by PCR from $P$. vivax with the F3 and B3 primers listed in Figure 1. The PCR products were purified with a Qiaquick gel extraction kit (QIAGEN, Inc., Valencia, CA, USA), and the eluted PCR products were cloned into a pGEM-T easy vector (Promega Corporation, Madison, WI, USA). The amplified DNA fragments were completely sequenced (Solgent Co., Ltd. Daejon, Korea) and confirmed to be identical to the sequence of the $\alpha$-tubulin gene from the $P$. vivax Sal-1 strain. The concentrations of plasmid DNA were measured with a NanoDrop ND-1000 spectrophotometer (NanoDrop Products, Thermo Scientific Instruments, Inc., Wilmington, DE, USA), and the corresponding copy numbers were calculated. The plasmid containing the $\alpha$ tubulin gene fragment was diluted with TE buffer (10 mM Tris- $\mathrm{HCl}, 1 \mathrm{mM}$ EDTA) to final concentrations of $1,10,10^{2}, 10^{3}$, or $10^{4}$ copies of the gene per reaction. The specificities of the LAMP assays were evaluated with genomic DNA from P. falciparum 3D7, P. vivax Sal-1, Plasmodium malariae Uganda I/CDC, Plasmodium ovale curtisi, Plasmodium ovale wallikeri, Plasmodium knowlesi H strain, Toxoplasma gondii RH, Cryptosporidium parvum Iowa, and Babesia microti Munich strains. The DNAs of the Entamoeba histolytica HM1:IMSS and Giardia lamblia WB strains were kindly provided by Dr. Myeong Heon Shin and Dr. Soon-Jung Park (Yonsei University College of Medicine, Seoul, Korea).

\section{Genomic DNA extraction from whole blood and nested PCR}

The DNAs were prepared from $100 \mu \mathrm{L}$ of whole blood with DNeasy tissue kits (QIAGEN, Inc.). One microlitre of extracted DNA that was dissolved in $20 \mu \mathrm{L}$ of doubledistilled water was used as a template for the nested PCR and LAMP assay. On the other hand, heat-treated lysates of whole blood samples were prepared as previously described with minor modification [36,37]. Briefly, $1 \mathrm{ml}$ of ice-cold $5 \mathrm{mM}$ sodium phosphate buffer ( $\mathrm{pH} 8.0$ ) was added to $20 \mu \mathrm{l}$ of whole blood sample, vortexed, and centrifuged at $12,000 \times g$ for $5 \mathrm{~min}$. The collected pellet was washed two times with $5 \mathrm{mM}$ sodium phosphate buffer ( $\mathrm{pH}$ 8.0), dissolved with $100 \mu \mathrm{l}$ of distilled water, and boiled for $10 \mathrm{~min}$. The samples were centrifuged again at $12,000 \times \mathrm{g}$ for $2 \mathrm{~min}$, and the supernatants were collected. Four microliter aliquots of the supernatants collected were used as templates for LAMP. The nested PCR based on the $18 \mathrm{~S}$ rDNA gene was performed as previously described [16] in triplicate with TaKaRa LA Taq polymerase (Takara Bio Inc., Shiga, Japan) in a reaction volume of $20 \mu \mathrm{L}$ with a thermal cycler (Perkin Elmer Cetus, PerkinElmer Inc., Waltham, MA, USA). Two primer sets for $18 \mathrm{~S}$ rDNA gene, the universal primer (P1, 5' $-\mathrm{AC}$ 
Table 2 Comparison of the results obtained with the diagnostic methods on the 177 whole blood samples

\begin{tabular}{|c|c|c|c|c|c|c|c|c|}
\hline Assay & No. positive & No. negative & $\begin{array}{l}\text { Sensitivity } \\
\left(95 \% \mathrm{Cl}^{\mathrm{d}}\right)\end{array}$ & $\begin{array}{l}\text { Specificity } \\
(95 \% \text { Cl) }\end{array}$ & $\begin{array}{l}\mathrm{PPV}^{\mathrm{a}} \\
(95 \% \mathrm{Cl})\end{array}$ & $\begin{array}{c}\mathrm{NPV}^{\mathrm{b}} \\
(95 \% \mathrm{Cl})\end{array}$ & $\begin{array}{c}\% \text { agreement with } \\
\text { nested PCR (Kappa) }\end{array}$ & $\begin{array}{c}A^{A U C} \\
(95 \% \mathrm{Cl})\end{array}$ \\
\hline Microscopy & 96 & 81 & $\begin{array}{c}75.0 \\
(66.8-81.7)\end{array}$ & $\begin{array}{c}100 \\
(91.1-100)\end{array}$ & $\begin{array}{c}100 \\
(95.3-100)\end{array}$ & $\begin{array}{c}60.5 \\
(49.6-70.4)\end{array}$ & $\begin{array}{c}81.9 \\
(0.624)\end{array}$ & $\begin{array}{c}0.875 \\
(0.817-0.920)\end{array}$ \\
\hline RDT & 137 & 40 & $\begin{array}{c}93.0 \\
(87.9-96.4)\end{array}$ & $\begin{array}{c}63.3 \\
(49.2-75.3)\end{array}$ & $\begin{array}{c}86.9 \\
(80.1-91.6)\end{array}$ & $\begin{array}{c}77.5 \\
(62.2-87.8)\end{array}$ & $\begin{array}{c}84.7 \\
(0.596)\end{array}$ & $\begin{array}{c}0.781 \\
(0.713-0.840)\end{array}$ \\
\hline Nested PCR & 128 & 49 & & & & & & \\
\hline a-tubulin LAMP & 137 & 40 & $\begin{array}{c}100 \\
(96.4-100)\end{array}$ & $\begin{array}{c}81.6 \\
(68.3-90.2)\end{array}$ & $\begin{array}{c}93.4 \\
(87.8-96.6)\end{array}$ & $\begin{array}{c}100 \\
(89.3-100)\end{array}$ & $\begin{array}{c}94.9 \\
(0.865)\end{array}$ & $\begin{array}{c}0.908 \\
(0.856-0.946)\end{array}$ \\
\hline
\end{tabular}

The sensitivities and specificities of the tests were determined using the nested PCR results as gold standard.

${ }^{a} \mathrm{PPV}$, positive predictive value, ${ }^{b} \mathrm{NPV}$, negative predictive value, ${ }^{c} \mathrm{AUC}$, area under the receiver operating characteristic curve, ${ }^{\mathrm{d}} \mathrm{Cl}$, confidence interval.

GATCAGATACCGTCGTAATCTT-3'; P2, 5' -GAACCCA AAGACTTTGATTTCTCAT-3') and the $P$. vivax specific primer (P1, 5'-ACGATCAGATACCGTCGTA ATCTT-3'; V1, 5' -CAATCTAAGAATAAACTCCGA AG AGAAA-3') that generated a 100 -bp product, were employed per reaction. The primer sets that were used had The PCR products from each reaction were confirmed by DNA sequencing (Solgent Co., Ltd.). The amplified products were visualized on $1.5 \%$ agarose gels, stained with ethidium bromide, observed with a UV transilluminator, and independently recorded by two experienced investigators blinded against the other methods.

\section{Statistical analysis}

The test sensitivities and specificities were determined using nested PCR as gold standard. The percentage sensitivities and specificities with 95\% confidence intervals (95\% CIs) were calculated with MedCalc, version 7.0 (MedCalc Software bvba, Ostend, Belgium). Differences in the sensitivities and specificities were compared with McNemar's normal approximation test with SAS 9.3 (SAS Institute, Inc., Cary, NC, USA). Differences between the areas under the receiver operating characteristic (AUC) were compared with a multiple logistic regression model and SAS 9.3 (SAS Institute, Inc.). The degree of agreement was determined using Kappa statistics in SAS 9.3 (SAS Institute Inc.). Statistical significance was accepted for $\mathrm{p}$ values less than 0.001 .

\section{Results}

\section{Optimization of the $P$. vivax a-tubulin LAMP assay conditions}

To develop a LAMP assay for the detection of $P$. vivax, a set of six primers targeting the $\alpha$-tubulin gene was designed (Figure 1). The targeting region of the $\alpha$-tubulin DNA in this LAMP assay showed relatively low sequence identity among Plasmodium spp. (P. knowlesi [GenBank accession no. XM_002258237]: 84.0\% nucleotide identity; P. falciparum, [GenBank accession no. XM_001351490]: $77.6 \%$ nucleotide identity)(Figure 1A). The optimal temperature and time for the LAMP reaction were determined with a cloned $\alpha$-tubulin gene fragment $\left(10^{6}\right.$ copies per reaction) under isothermal conditions at temperatures of $60^{\circ} \mathrm{C}$ to $65^{\circ} \mathrm{C}$ for $120 \mathrm{~min}$ by monitoring turbidity. Although amplification targeting of the $P$. vivax $\alpha$-tubulin gene was detected at all of the temperatures tested, a threshold value of absorbance (0.1) from the LAMP assay was reached most quickly at $64^{\circ} \mathrm{C}$ (data not shown). No nonspecific amplification was detected in the negative control (plasmid containing no insert) after at least $120 \mathrm{~min}$ of incubation. Thus, the subsequent LAMP reactions were conducted at $64^{\circ} \mathrm{C}$ for $90 \mathrm{~min}$.

\section{Analytical sensitivity and specificity of the $P$. vivax a-tubulin LAMP assay}

To assess the sensitivity of the $\alpha$-tubulin LAMP assay, the LAMP assay was conducted with serially diluted plasmid DNAs containing the $\alpha$-tubulin gene to the equivalent of $10^{5}$ to 1 copy per reaction. The LAMP procedure amplified the targeted region at each dilution from the highest copy numbers $\left(10^{5}\right.$ copies per reaction) (Figure 2B) to as little as 100 copies of $P$. vivax $\alpha-$ tubulin gene per reaction (linear regression coefficient $\left.\mathrm{R}^{2}=0.9579\right)$, which reached the threshold value of $\mathrm{ab}$ sorbance (0.1) within $50 \mathrm{~min}$ (Figure 2A and B). The specificity of the $P$. vivax $\alpha$-tubulin LAMP assay was evaluated with the genomic DNA of five known malariaassociated species ( $P$. falciparum, P. malariae, P. ovale curtisi, P. ovale wallikeri, P. knowlesi) and seven pathogenic but malaria-unrelated species, three belonging to the phylum Apicomplexa (Babesia microti, Toxoplasma gondii, and Cryptosporidium parvum) and four protozoan parasites (Entamoeba histolytica, Giardia lamblia, Trichomonas vaginalis, and Acanthamoeba castellanii). As shown in Figure 2C, a typical ladder pattern of the amplified LAMP products with agarose gel electrophoresis was only observed for the $P$. vivax genomic DNA. The $P$. vivax $\alpha$-tubulin LAMP assay showed no detectable amplification of the other DNAs, including the malaria-negative human DNA controls (Figure 2C). The amplified products of the positive reactions were also visualized with fluorescent detection reagents under UV 

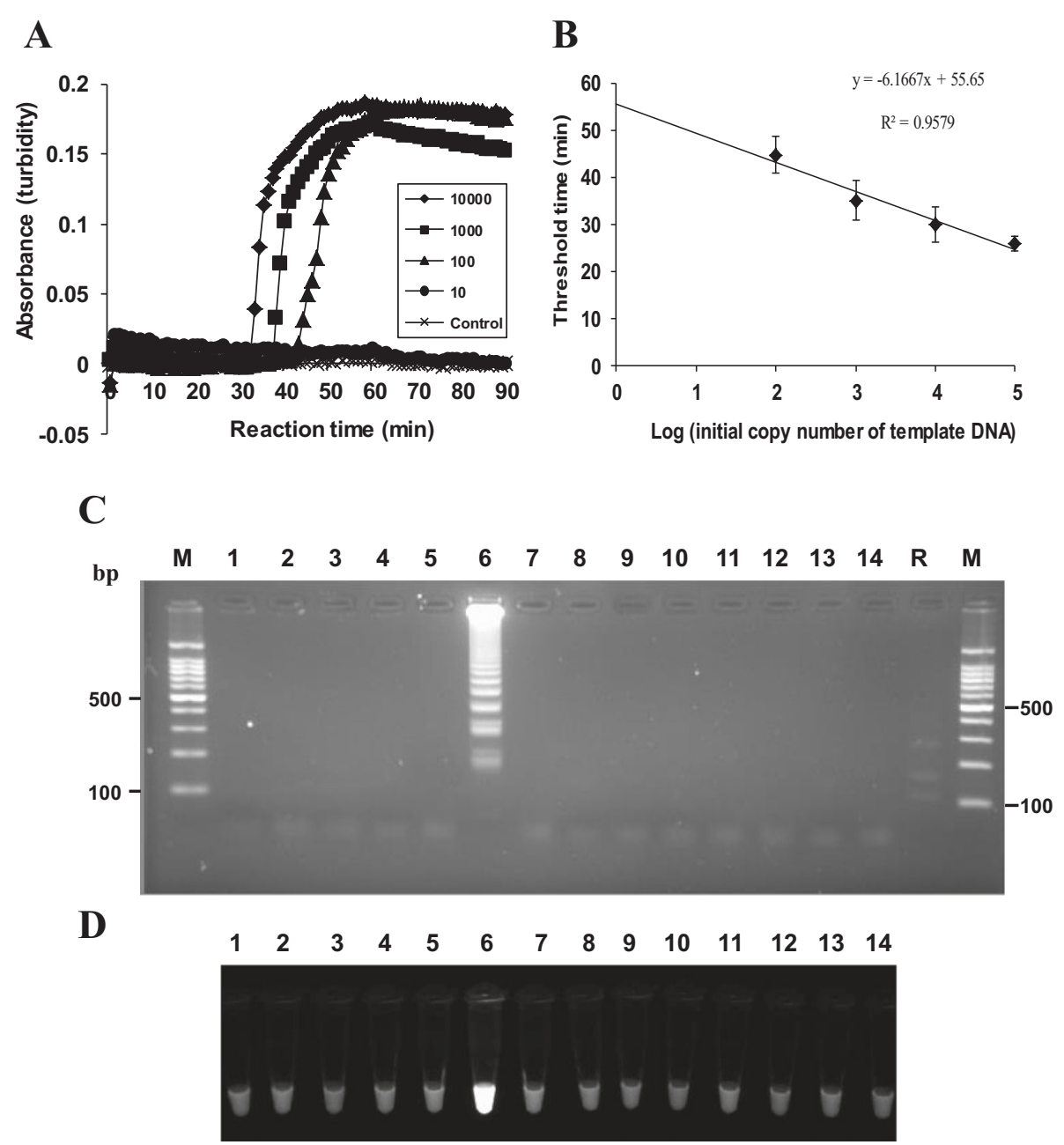

Figure 2 Sensitivity and specificity of the $P$. vivax a-tubulin LAMP assay. (A) Sensitivity of the $P$. vivax a-tubulin LAMP assay. Serial 10-fold dilutions of the a-tubulin DNA $\left(10,10^{2}, 10^{3}\right.$, and $10^{4}$ copies per reaction) were used for the LAMP assay, and the real-time amplification was monitored by a measurement of absorbance. (B) Correlation between the threshold time and the copy number of the a-tubulin DNA in serially diluted samples. The values on the $y$-axis are the threshold time (in min), which was defined as the time at which the threshold value of absorbance (0.1) was reached. The results show the means and standard deviations of three separate experiments. A plasmid containing no insert was used as a control. The LAMP products were visualized using (C) gel electrophoresis and (D) Loopamp fluorescent detection reagent (FD). Lane M, a 100-bp molecular weight marker; lane 1, P. falciparum; lane 2, Plasmodium ovale curtisi; lane 3, Plasmodium ovale wallikeri; lane 4, Plasmodium malariae; lane 5, Plasmodium knowlesi; lane 6, P. vivax; lane 7, Babesia microti; lane 8, Toxoplasma gondii; lane 9, Cryptosporidium parvum; lane 10, Entamoeba histolytica; lane 11, Giardia lamblia; lane 12, Trichomonas vaginalis; lane 13; Acanthamoeba castellanii; lane 14, malaria-negative human DNA control; lane R, products of the Aatll digestion of the LAMP product of a-tubulin.

light (Figure 2D). In order to determine the specificity of the amplification by the $\alpha$-tubulin LAMP assay, the amplified LAMP products from the $P$. vivax genomic DNA were digested with AatII (Figure 1, arrowhead) and found to generate the expected 187- and 137-bp fragments (Figure 2C, lane R).

\section{Validation of the $P$. vivax a-tubulin LAMP assay}

To validate the $\alpha$-tubulin LAMP assays, the blood samples of 177 male ROK soldiers who were admitted with febrile illness and who were suspected of having malaria were subjected to $\alpha$-tubulin LAMP and three available methods, including microscopic examinations, immunochromatographic RDTs and nested PCRs. To determine the accuracy of the tests for diagnosis of $P$. vivax malaria, nested PCR was used as a gold standard and the sensitivity, specificity, positive predictive value (PPV), and negative predictive value (NPV) were calculated for each test. Of the 177 male ROK soldiers, 96 (54\%), 137 (77\%), 128 (72\%), and 137 (77\%) were positive for microscopy, RDT, nested PCR, and $\alpha$-tubulin LAMP, respectively. Microscopic examinations were positive for 96 specimens, resulting in a sensitivity of $75.0 \%$ (95\% CI, $66.8 \%-81.7 \%)$ and a specificity of $100 \%$ (95\% CI, $91.1 \%$ - 
100\%) (Table 2). The median parasite density in the 96 male patients was $1,263[103-5,177] / \mu \mathrm{L}$. All 96 positive specimens by microscopy were nested PCR positives. Thirty-two of the 81 negative specimens by microscopy were true positives by nested PCR and thus considered false negatives (Table 1). The results obtained by RDT were positive for 137 specimens, resulting in a sensitivity of $93.0 \%$ (95\% CI, $87.9 \%-96.4 \%)$ and a specificity of $63.3 \%$ (95\% CI, 49.2\%-75.3\%) (Table 2). Eighteen of the 137 positive $(18 / 137,13.1 \%)$ by RDT were negative by nested PCR for the detection of $P$. vivax and thus considered false positives. Nine of the 40 specimens $(9 / 40$, $22.5 \%$ ) negative by RDT were true positive by nested PCR and thus considered false negatives (Table 1). All malaria-positive cases by RDT were identified as $P$. vivax infections.

The sensitivity of the $\alpha$-tubulin LAMP assays was $100 \%$ (95\% CI, 96.4\%-100\%), and its specificity was 81.6\% (95\% CI, 68.3\%-90.2\%) (Table 2). Eight that were positive by RDT but negative by nested PCR were positive by $\alpha$-tubulin LAMP (Table 1). Of the 137 positives by $\alpha$-tubulin LAMP, 128 specimens (136/137, 99.3\%) were true positives, and thus 9 specimens were considered a false positive (Table 1). Ten of the 40 negative specimens by $\alpha$-tubulin LAMP assays were only positive by RDT. On the other hand, $\alpha$-tubulin LAMP assay conducted using heat-treated lysates produced the same results as those obtained using a commercial kit (DNeasy tissue kit) (data not shown). When nested PCR was used as the gold standard, the $\alpha$-tubulin LAMP assays had the highest diagnostic sensitivity $(P<0.001$ vs. microscopy; $P=0.0023$ vs. RDT) and better agreement (concordance: $94.9 \%$, kappa value: 0.865$)$ with nested PCR than RDT and microscopy (Table 2). To compare the diagnostic accuracies of microscopy, RDT, and $\alpha$-tubulin LAMP assays for the detection of $P$. vivax, the diagnostic accuracies were assessed by performing ROC analyses and measuring the AUCs. Among these three diagnostic tests when nested PCR was used as the gold standard, the $\alpha$-tubulin LAMP assay had the highest AUC (0.908) $(P=0.4272$ vs. microscopy; $P=0.0104$ vs. RDT) among the three tests.

\section{Discussion}

The resurgence of vivax malaria in many parts of the world emphasizes the need for a rapid, sensitive, and inexpensive diagnostic method. Furthermore, it needs to be easily performed and to produce readily interpretable results in the field of an endemic area. As one of the diagnostic methods of vivax malaria, microscopic examinations of blood samples are still widely used in many malaria-endemic areas, including the ROK. As shown in Table 2, microscopic examinations had the lowest sensitivity $(75.0 \%)$ among the four tests in this study.
Furthermore, the requirement of well-trained experts and the long turnaround time limit its usefulness in the field. Due to its convenience, RDT is currently and widely used as an alternative field diagnostic test. However, recent studies suggest that RDT had relatively lower sensitivity and specificity for $P$. vivax than $P$. falciparum $[14,15,38]$. The RDT in this study had high sensitivity relative to that of the microscopy, and had lower specificity and negative predictive values compared to those of $\alpha$-tubulin LAMP assays by considering nested PCR as gold standard (Table 2). To solve these problems, molecular diagnostic tests, nested PCRs, and LAMP assays have been developed [11,39]. In particular, $P$. vivax LAMP assays that are based on $18 \mathrm{~S} \mathrm{rDNA}$, mitochondrial DNA, and Pvr64 have recently been developed and validated for the diagnosis of malaria [28-30,40-44]. The sensitivities of nested PCRs and LAMP assays for $P$. falciparum malaria have been shown to be comparable or higher than RDTs, respectively [45]. Thus, the aim of this study was to design and test the utility of a novel set of $P$. vivax-specific LAMP primers with different target DNA sequences in order to improve diagnostic performance and validate them as an alternative molecular diagnostic test. Although the tubulin gene of $P$. vivax is not species-specific, the $\beta$-tubulin gene sequence has previously been used successfully to amplify P. knowlesi DNA with a LAMP assay [46]. The targeting region of the $\alpha$-tubulin DNA in this LAMP assay showed relatively low sequence identity among Plasmodium spp. and its use has resulted in no cross reactivity with the other malaria species examined.

In the present study, the diagnostic performance of $\alpha$ tubulin LAMP assays were compared to three currently available tests, including microscopic examinations, RDT, and nested PCRs using 177 whole blood specimens. When nested PCR was used as gold standard, 128 (72.3\%) of the 177 specimens were positive for vivax malaria. PCR is still the most sensitive and specific method for vivax malaria among all of the currently available tests [47]. However, expensive laboratory equipment, the need for a trained specialist, and timeconsuming post-PCR procedures, such as agarose gel electrophoresis, limit the usefulness of nested PCR in the field when diagnostic equipment is minimal. However, a LAMP assay does not require a thermal cycler and can be performed with minimal laboratory facilities, such as a heating block or a water bath, and its end points can be determined by turbidity or color development by eye. Furthermore, the straightforward sample preparation for molecular diagnosis reduces the risk of cross-contamination, and thus the risks posed by falsepositive results. LAMP assays that are based merely on heat-treated blood have previously been found to be as efficient at detecting malaria parasites as DNA that is 
extracted with a commercial kit $[44,48]$. In this study, results of $\alpha$-tubulin LAMP assay based on heat-treated samples were consistent with those of the LAMP assay using a commercial kit. Thus, the devised P. vivax LAMP assay could eliminate the need for DNA extraction without compromising sensitivity and reduce the time required to reach a diagnosis. The $\alpha$-tubulin LAMP assay had the higher diagnostic accuracy $(\mathrm{AUC}=0.908$ ) than microscopy and RDT and showed better agreement with nested PCR (Table 2) and thus can be used in the diagnosis of early infections of vivax malaria as an alternative molecular diagnostic tool.

In endemic countries, including the ROK, vivax malaria is also transmitted by the transfusion of infected blood products $[49,50]$, and, as yet, no reliable approved laboratory test is available for the screening of donated blood. Although the LAMP assay was applied to whole blood samples of patients suspected to be infected, the LAMP assay devised here could also be used for blood screening for $P$. vivax. Furthermore, individuals with low parasite density can provide reservoirs for transmission, and thus an early and accurate diagnostic tool like the $\alpha$-tubulin LAMP assay may help to prevent transmission in endemic areas.

\section{Conclusion}

This study describes about the development and validation of a LAMP assay for the detection of $P$. vivax DNA in clinical blood samples. In addition, the $P$. vivax $\alpha$-tubulin LAMP assay developed here can be used to diagnose early infections of vivax malaria, is an alternative molecular diagnostic tool and a point-of-care test that may help to prevent transmission in endemic areas.

\section{Competing interests}

The authors declare that they have no competing interests.

\section{Authors' contributions \\ $\mathrm{BKN}, \mathrm{NI}, \mathrm{HHK}, \mathrm{DIC}, \mathrm{YKG}$ and $\mathrm{YH}$ conceived and designed the study and contributed to the execution of the research. SD and YH wrote the manuscript. WKL contributed statistical analysis. SJ collected the blood samples in the field. SD and HWY carried out the molecular diagnostic tests. HWY, SYJ and SJ performed microscopic examination and RDT. All authors have read and approved the final manuscript.}

\section{Acknowledgements}

This research was supported by the Basic Science Research Program through the National Research Foundation of Korea funded by the Ministry of Education, Science and Technology (NRF-2010-0016679). We wish to express our appreciation to Dr. Kawai Satoru (Dokkyo Medical University) for providing P. knowlesi genomic DNA. We are indebted to Dr. Jung-Yeon Kim (The Korea Centers for Disease Control and Prevention) for providing the $P$. malariae and P. ovale DNA.

\section{Author details}

'Department of Parasitology and Tropical Medicine, Kyungpook National University School of Medicine, 700-422 Daegu, Republic of Korea. 2Department of Medicine, ROK Army Headquarters, Choongnam, Republic of Korea. ${ }^{3}$ Department of Parasitology and Institute of Health Sciences, Gyeongsang National University School of Medicine, 660-751 Jinju, Republic of Korea. ${ }^{4}$ National Research Center for Protozoan Diseases, Obihiro
University of Agriculture and Veterinary Medicine, 080-8555 Obihiro, Japan. ${ }^{5}$ Department of Preventive Medicine, Kyungpook National University Medical Center, Kyungpook National University School of Medicine, Daegu, Republic of Korea. ${ }^{6}$ Department of Parasitology, Dong-A University, College of Medicine, 602-714, Busan, Republic of Korea.

Received: 7 March 2014 Accepted: 26 June 2014

Published: 30 June 2014

\section{References}

1. Guerra CA, Howes RE, Patil AP, Gething PW, Van Boeckel TP, Temperley WH, Kabaria CW, Tatem AJ, Manh BH, Elyazar IR, Baird JK, Snow RW, Hay SI: The international limits and population at risk of Plasmodium vivax transmission in 2009. PloS Negl Trop Dis 2010, 4:e774.

2. Galinski MR, Barnwell JW: Plasmodium vivax: who cares? Malar J 2008, 7(Suppl 1):S9.

3. Mueller I, Galinski MR, Baird JK, Carlton JM, Kochar DK, Alonso PL, del Portillo HA: Key gaps in the knowledge of Plasmodium vivax, a neglected human malaria parasite. Lancet Infect Dis 2009, 9:555-566.

4. Price RN, Tjitra E, Guerra CA, Yeung S, White NJ, Anstey NM: Vivax malaria: neglected and not benign. Am J Trop Med Hyg 2007, 77:79-87.

5. Kochar DK, Saxena V, Singh N, Kochar SK, Kumar SV, Das A: Plasmodium vivax malaria. Emerg Infect Dis 2005, 11:132-134.

6. Genton B, D'Acremont V, Rare L, Baea K, Reeder JC, Alpers MP, Muller I: Plasmodium vivax and mixed infections are associated with severe malaria in children: a prospective cohort study from Papua New Guinea. PLoS Med 2008, 5:e127.

7. Chai IH, Lim Gl, Yoon SN, Oh WI, Kim SJ, Chai JY: [Occurrence of tertian malaria in a male patient who has never been abroad]. Korean J Parasitol 1994, 32:195-200.

8. Park JW, Jun G, Yeom JS: Plasmodium vivax malaria: status in the Republic of Korea following reemergence. Korean J Parasitol 2009, 47(Suppl):S39-50.

9. Reyburn H: New WHO guidelines for the treatment of malaria. BMJ 2010, 340:c2637.

10. Bruce-Chwatt L: From Laveran's discovery to DNA probes: new trends in diagnosis of malaria. Lancet 1987, 2:1509-1511.

11. Moody A: Rapid diagnostic tests for malaria parasites. Clin Microbiol Rev 2002, 15:66-78.

12. Barker RH Jr: DNA probe diagnosis of parasitic infections. Exp Parasitol 1990, 70:494-499.

13. WHO: Malaria rapid diagnostic test performance: Results of WHO product testing of malaria RDTs Round 2 (2009). Geneva, Switzerland: WHO; 2009.

14. Mens P, Spieker N, Omar S, Heijnen M, Schallig H, Kager PA: Is molecular biology the best alternative for diagnosis of malaria to microscopy? A comparison between microscopy, antigen detection and molecular tests in rural Kenya and urban Tanzania. Trop Med Int Health 2007, 12:238-244.

15. Murray CK, Gasser RA Jr, Magill AJ, Miller RS: Update on rapid diagnostic testing for malaria. Clin Microbiol Rev 2008, 21:97-110.

16. Kimura M, Kaneko O, Liu Q, Zhou M, Kawamoto F, Wataya Y, Otani S, Yamaguchi $Y$, Tanabe $K$ : Identification of the four species of human malaria parasites by nested PCR that targets variant sequences in the small subunit rRNA gene. Parasitol Int 1997, 46:91-95.

17. Perandin F, Manca N, Calderaro A, Piccolo G, Galati L, Ricci L, Medici MC, Arcangeletti MC, Snounou G, Dettori G, Chezzi C: Development of a realtime PCR assay for detection of Plasmodium falciparum, Plasmodium vivax, and Plasmodium ovale for routine clinical diagnosis. J Clin Microbiol 2004, 42:1214-1219.

18. Rougemont M, Van Saanen M, Sahli R, Hinrikson HP, Bille J, Jaton K: Detection of four Plasmodium species in blood from humans by $18 \mathrm{~S}$ rRNA gene subunit-based and species-specific real-time PCR assays. J Clin Microbiol 2004, 42:5636-5643.

19. Singh B, Bobogare A, Cox-Singh J, Snounou G, Abdullah MS, Rahman HA: A genus- and species-specific nested polymerase chain reaction malaria detection assay for epidemiologic studies. Am J Trop Med Hyg 1999, 60:687-692.

20. Snounou G, Viriyakosol S, Jarra W, Thaithong S, Brown KN: Identification of the four human malaria parasite species in field samples by the polymerase chain reaction and detection of a high prevalence of mixed infections. Mol Biochem Parasitol 1993, 58:283-292.

21. Snounou G, Viriyakosol S, Zhu XP, Jarra W, Pinheiro L, do Rosario VE, Thaithong S, Brown KN: High sensitivity of detection of human malaria 
parasites by the use of nested polymerase chain reaction. Mol Biochem Parasitol 1993, 61:315-320.

22. Hanscheid T, Grobusch MP: How useful is PCR in the diagnosis of malaria? Trends Parasitol 2002, 18:395-398.

23. Notomi T, Okayama H, Masubuchi H, Yonekawa T, Watanabe K, Amino N, Hase T: Loop-mediated isothermal amplification of DNA. Nucleic Acids Res 2000, 28:E63.

24. Mori Y, Notomi T: Loop-mediated isothermal amplification (LAMP): a rapid, accurate, and cost-effective diagnostic method for infectious diseases. J Infect Chemother 2009, 15:62-69.

25. Mori Y, Nagamine K, Tomita N, Notomi T: Detection of loop-mediated isothermal amplification reaction by turbidity derived from magnesium pyrophosphate formation. Biochem Biophys Res Commun 2001, 289:150-154.

26. Goto M, Honda E, Ogura A, Nomoto A, Hanaki K: Colorimetric detection of loop-mediated isothermal amplification reaction by using hydroxy naphthol blue. Biotechniques 2009, 46:167-172.

27. Tomita N, Mori Y, Kanda H, Notomi T: Loop-mediated isothermal amplification (LAMP) of gene sequences and simple visual detection of products. Nat Protoc 2008, 3:877-882.

28. Han ET, Watanabe R, Sattabongkot J, Khuntirat B, Sirichaisinthop J, Iriko H, Jin L, Takeo S, Tsuboi T: Detection of four Plasmodium species by genusand species-specific loop-mediated isothermal amplification for clinical diagnosis. J Clin Microbiol 2007, 45:2521-2528.

29. Tao ZY, Zhou HY, Xia H, Xu S, Zhu HW, Culleton RL, Han ET, Lu F, Fang Q, Gu YP, Liu YB, Zhu GD, Wang WM, Li JL, Cao J, Gao Q: Adaptation of a visualized loop-mediated isothermal amplification technique for field detection of Plasmodium vivax infection. Parasit Vectors 2011, 4:115.

30. Patel JC, Oberstaller J, Xayavong M, Narayanan J, DeBarry JD, Srinivasamoorthy G, Villegas L, Escalante AA, DaSilva A, Peterson DS, Barnwell JW, Kissinger JC, Udhayakumar V, Lucchi NW: Real-time loop-mediated isothermal amplification (RealAmp) for the species-specific identification of Plasmodium vivax. PLoS One 2013, 8:e54986.

31. Karanis $P$, Ongerth J: LAMP-a powerful and flexible tool for monitoring microbial pathogens. Trends Parasitol 2009, 25:498-499.

32. DeLong ER, DeLong DM, Clarke-Pearson DL: Comparing the areas under two or more correlated receiver operating characteristic curves: a nonparametric approach. Biometrics 1988, 44:837-845.

33. Campuzano-Zuluaga G, Hanscheid T, Grobusch MP: Automated haematology analysis to diagnose malaria. Malar J 2010, 9:346.

34. Iwagami M, Fukumoto M, Hwang S-Y, Kim S-H, Kho W-G, Kano S: Population structure and transmission dynamics of Plasmodium vivax in the Republic of Korea based on microsatellite DNA analysis. PLoS Negl Trop Dis 2012, 6:e1592.

35. PrimerExplorer V4. [http://primerexplorer.jp/e/]

36. Foley M, Ranford-Cartwright LC, Babiker HA: Rapid and simple method for isolating malaria DNA from fingerprick samples of blood. Mol Biochem Parasitol 1992, 53:241-244.

37. Henning L, Felger I, Beck H-P: Rapid DNA extraction for molecular epidemiological studies of malaria. Acta Trop 1999, 72:149-155.

38. Wongsrichanalai C, Barcus MJ, Muth S, Sutamihardja A, Wernsdorfer WH: A review of malaria diagnostic tools: microscopy and rapid diagnostic test (RDT). Am J Trop Med Hyg 2007, 77:119-127.

39. Abdul-Ghani R, Al-Mekhlafi AM, Karanis P: Loop-mediated isothermal amplification (LAMP) for malarial parasites of humans: would it come to clinical reality as a point-of-care test? Acta Trop 2012, 122:233-240.

40. Pöschl B, Waneesorn J, Thekisoe O, Chutipongvivate S, Panagiotis K: Comparative diagnosis of malaria infections by microscopy, nested PCR, and LAMP in northern Thailand. Am J Trop Med Hyg 2010, 83:56.

41. Chen J-H, Lu F, Lim CS, Kim J-Y, Ahn H-J, Suh I-B, Takeo S, Tsuboi T, Sattabongkot J, Han E-T: Detection of Plasmodium vivax infection in the Republic of Korea by loop-mediated isothermal amplification (LAMP). Acta Trop 2010, 113:61-65.

42. Sirichaisinthop J, Buates S, Watanabe R, Han E-T, Suktawonjaroenpon W, Krasaesub S, Takeo S, Tsuboi T, Sattabongkot J: Short Report: Evaluation of Loop-Mediated Isothermal Amplification (LAMP) for Malaria Diagnosis in a Field Setting. Am J Trop Med Hyg 2011, 85:594-596.

43. Polley SD, González IJ, Mohamed D, Daly R, Bowers K, Watson J, Mewse E, Armstrong M, Gray C, Perkins MD: Clinical evaluation of a loop-mediated amplification kit for diagnosis of imported malaria. J Infect Dis 2013, 208:637-644.

44. Polley SD, Mori Y, Watson J, Perkins MD, González IJ, Notomi T, Chiodini PL, Sutherland CJ: Mitochondrial DNA targets increase sensitivity of malaria detection using loop-mediated isothermal amplification. J Clin Microbiol 2010, 48:2866-2871.

45. Lee PW, Ji DD, Liu CT, Rampao HS, Do Rosario VE, Lin IF, Shaio MF: Application of loop-mediated isothermal amplification for malaria diagnosis during a follow-up study in Sao Tome. Malar J 2012, 11:408.

46. Iseki H, Kawai S, Takahashi N, Hirai M, Tanabe K, Yokoyama N, Igarashi I: Evaluation of a loop-mediated isothermal amplification method as a tool for diagnosis of infection by the zoonotic simian malaria parasite Plasmodium knowlesi. J Clin Microbiol 2010, 48:2509-2514.

47. WHO: Malaria Diagnosis: New Perspectives. Geneva, Switzerland: WHO; 2000

48. Poon LL, Wong BW, Ma EH, Chan KH, Chow LM, Abeyewickreme W, Tangpukdee N, Yuen KY, Guan Y, Looareesuwan S: Sensitive and inexpensive molecular test for falciparum malaria: detecting Plasmodium falciparum DNA directly from heat-treated blood by loop-mediated isothermal amplification. Clin Chem 2006, 52:303-306.

49. Mungai M, Tegtmeier G, Chamberland M, Parise M: Transfusion-transmitted malaria in the United States from 1963 through 1999. N Engl J Med 2001, 344:1973-1978.

50. Oh JS, Kim JS, Lee CH, Nam DH, Kim SH, Park DW, Lee CK, Lim CS, Park GH: Evaluation of a malaria antibody enzyme immunoassay for use in blood screening. Mem Inst Oswaldo Cruz 2008, 103:75-78.

doi:10.1186/1475-2875-13-248

Cite this article as: Dinzouna-Boutamba et al:: The development of loopmediated isothermal amplification targeting alpha-tubulin DNA for the rapid detection of Plasmodium vivax. Malaria Journal 2014 13:248.

\section{Submit your next manuscript to BioMed Central and take full advantage of:}

- Convenient online submission

- Thorough peer review

- No space constraints or color figure charges

- Immediate publication on acceptance

- Inclusion in PubMed, CAS, Scopus and Google Scholar

- Research which is freely available for redistribution 